\title{
Adaptación de un cuestionario para evaluar las creencias epistemológicas sobre la matemática de profesores de secundaria básica
}

\section{Adaptation of a questionnaire to assess the epistemological beliefs of mathematics in secondary school teachers}

\author{
Annia VIZCAINO ESCOBAR ${ }^{1}$, Arianys CADALSO ROMERO ${ }^{1} \mathrm{y}$ \\ Mayra MANZANO MIER ${ }^{2}$ \\ ${ }^{1}$ Universidad Central “Marta Abreu” de Las Villas y ${ }^{2}$ Universidad de La Habana.
}

Recibido: Septiembre 2013

Aceptado: Noviembre 2013

\begin{abstract}
Resumen
El objetivo del estudio está orientado a determinar las propiedades psicométricas del Cuestionario de Creencias Epistemológicas sobre la Matemática. Participan 171 profesores de matemática de Secundaria Básica de la Región Central de Cuba. Los resultados muestran una consistencia interna aceptable. La estructura factorial de la escala reveló tres factores principales, coherentes con el modelo de los tres constructos: creencias sobre el conocimiento, sobre el aprendizaje y la enseñanza, mostrando niveles irregulares en el desarrollo del sistema de creencias epistemológicas sobre la matemática de estos docentes que tienden a los polos de ingenuidad y sofisticación. En conclusión, el cuestionario es útil para evaluar las creencias docentes sobre la matemática.
\end{abstract}

Palabras clave: adaptación, cuestionario, creencias epistemológicas sobre la matemática, profesores, secundaria básica.

\begin{abstract}
The objective of the study is to determine the psychometric properties of the Epistemological Beliefs Questionnaire on Mathematics. 171 Secondary School Mathematics Teachers of the Central Region of Cuba participated. The results show acceptable internal consistency. The factorial structure of the scale revealed three major factors, consistent with the Model of the Three Constructs: beliefs about knowledge, about learning and teaching. Irregular levels in the development of the epistemological belief system about mathematics of these teachers were shown, with a tendency among naivety and sophistication poles. In conclusion, the questionnaire is useful for evaluating teacher's beliefs about mathematics.
\end{abstract}

Keywords: adaptation, questionnaire, epistemological beliefs about mathematics, teachers, junior high. 
El término “epistemología” tiene su origen en la filosofía, sin embargo, ha sido utilizado en el estudio que por décadas se ha realizado sobre las creencias que tienen los protagonistas del proceso de enseñanza y aprendizaje, sobre el origen y la adquisición del conocimiento (Hofer, 2001). Esta misma autora define la epistemología como lo concerniente al origen, naturaleza, límites, métodos y justificación del conocimiento humano; constituye además un set identificable de dimensiones de creencias sobre el conocimiento y cómo se conoce o aprende, que está organizado como teorías que progresan en direcciones predictivas y que se activan con el contexto, operando entre lo cognitivo y lo metacognitivo (Hofer, 2004).

El estudio sobre la epistemología personal comienza en los EE.UU y ha evolucionado tras décadas de estudio expandiéndose por diferentes culturas (Hofer, 2008). Esta extensión a otros contextos ha aportado sustanciosos resultados complementando las investigaciones iniciales del tema.

Dentro de esta perspectiva han existido varios modelos y propuestas (Conley \& Harrison, 2004). Los cuales han oscilado desde modelos que proponen una dimensión general del pensamiento epistemológico (Perry, 1970) que evoluciona con el tiempo en su forma de comportarse; a modelos que proponen algún número finito de dimensiones, usualmente de 4 a 7 (Schommer, 1990; Hofer \& Pintrich, 1997) a otros que proponen muchas dimensiones diferentes de los recursos epistemológicos (Hammer \& Elby, 2002). Pintrich (2002) reconoce la falta de consenso sobre el número de dimensiones en la investigación sobre el tema, pero sugirió que los modelos que proponen un número finito de dimensiones pueden ofrecer el mejor acuerdo.

Hoffer (2008) refiere que las dos categorías más estudiadas respecto a la epistemología personal es precisamente el desarrollo epistémico y las creencias epistemológicas. Schommer (1990) propuso una nueva conceptualización de las creencias y un enfoque teórico diferente al que se había desarrollado anteriormente, el cual fue llamado en la literatura como el Paradigma Multidimensional. Dicho paradigma modifica la noción unidimensional de la epistemología personal que por años había persistido y presenta un enfoque más complejo, abierto y flexible de las creencias epistemológicas.

Con el modelo que propone Marlene Schommer (1990) se asume un número de dimensiones de las creencias que puede variar, que constituye un sistema donde cada una de ellas está relacionada pero no atada con las demás, constituyendo un sistema más o menos independiente cuyo desarrollo no es necesariamente sincrónico. Fueron definidas como dimensiones la fuente de la autoridad, la estructura y la certeza del conocimiento, la velocidad del aprendizaje y la habilidad para aprender, (Shommer, 1994). Todas son vistas en un continuo que va desde la visión superficial o ingenua que ella llamó “simple” hasta la visión más compleja que pondera las variables que la autora denominó como “sofisticada” que tienen las personas con mayor desarrollo.

Siguiendo estas ideas, se reconoce el valor de la epistemología personal y sus múltiples variables ya que al construir una epistemología personal, los individuos se involucran activamente en el aprendizaje; persisten en las tareas difíciles; comprenden 
el material escrito y abordan temas poco estructurados, habilidades que se requieren para un mejor aprendizaje tanto de alumnos como de profesores. Además las creencias epistemológicas son premisas a tomar en cuenta ya que determinan las acciones pedagógicas y de aprendizaje de alumnos y profesores. Estas creencias pueden apoyar la motivación y la disposición de los alumnos para aprender y de los profesores por facilitar al aprendizaje, o pueden actuar como una barrera que lo dificulte (Gómez \& Silas, 2012).

En distintas investigaciones se ha comprobado la relación existente entre las variables del aprendizaje y las creencias epistemológicas (Cano, 2005; Conley \& Harrison, 2004; Mendoza, 2012; Schommer, 1990; Schommer-Aikins, 2008); en algunas se ha evidenciado la relación con variables cognitivas como el pensamiento, específicamente su influencia en el juicio reflexivo que se puede manifestar en la comprensión de textos y el monitoreo de este proceso de comprensión (Schommer, 1990); en otros para la interpretación de temas controvertidos, la solución de problemas mal definidos, en la transferencia del aprendizaje y el cambio conceptual (Manson, 2004).

Un ejemplo de la importancia de las creencias para el estudio del aprendizaje ha sido comprobado por Duell y Schommer-Aikins (2001) quienes encontraron que mientras más simple, manejable, cierto y dependiente de la autoridad, el alumno consideraba el conocimiento, expresaban mayor tendencia a simplificar la información. Probablemente uno de los elementos que ha influido decisivamente en la relevancia de las creencias epistemológicas en el contexto educativo ha sido estas relaciones entre estas variables cognitivas y del aprendizaje.

A través de estas aproximaciones al tema de la epistemología personal, se reconoce la necesidad de indagar en el proceso de formación de las creencias en los alumnos y cómo se hacen sensibles a los aspectos epistemológicos de su ambiente instruccional; cómo se da el vínculo entre sus creencias epistemológicas, las prácticas pedagógicas y las creencias de los profesores como mediadoras de los procesos de enseñanza (Hofer, 2001). Estos antecedentes nos convocan a varios retos, entre ellos, al análisis de las creencias epistemológicas de los profesores, a determinar cómo influyen en el diseño de la profesión pedagógica, en los enfoques y métodos de enseñanza que utilizan, en la formación de las creencias de sus alumnos.

\section{Creencias epistemológicas de los docentes}

Las creencias de los docentes son estudiadas con el fin de comprender e interpretar las acciones del profesor y asimismo promover cambios que transformen a su vez las prácticas docentes. Las investigaciones acerca de las creencias epistemológicas de los docentes se han desarrollado en diferentes escenarios (Chan, 2005; Ching \& Swe, 2008; García \& C., 2011; Inguanzo, 2010) y han sido evaluadas a partir del paradigma del pensamiento del profesor. Este paradigma ve al profesor como un sujeto reflexivo, racional, que toma decisiones, emite juicios, tiene creencias y genera rutinas propias de su desarrollo profesional. Desde este enfoque se tiende a la caracterización de las 
creencias sobre el conocimiento, el aprendizaje y la enseñanza en los profesores (Prieto, 2008; Serrano, 2010).

Aunque las creencias son una parte relevante en la vida profesional de los docentes ya que operan como la base que guía sus acciones y tiene un componente motivacional implícito, éstas son una arista menos explorada en el tema de la epistemología personal. La posibilidad de cambiar la enseñanza sin cambiar las creencias que a ésta subyacen parece difícil, pues sin conciencia de las razones que alientan la práctica docente resulta complejo modificar aspectos de la misma con el fin de mejorarla (Prieto, 2008).

Entre los aspectos sobre los cuales influyen las creencias docentes encontramos: la implementación del currículo; los enfoques sobre la enseñanza y las estrategias docentes; los esfuerzos para realizar adaptaciones curriculares; la flexibilidad para considerar enfoques alternativos de enseñanza; por solo citar algunos (Aguilar, 2012).

Siguiendo estas ideas el estudio de las creencias docentes permite comprender, explicar, renovar la dinámica del proceso enseñanza-aprendizaje, no obstante consideramos necesario avanzar aún más en su definición teórica.

\section{Definiendo las creencias epistemológicas de los profesores}

Conceptualizar las creencias epistemológicas de los profesores separadas de las investigaciones previas que se han realizado sobre este tipo de creencias en estudiantes, ha constituido un problema en la epistemología personal (Schraw, Bendixen, \& Dunkle, 2002). Un mejor acercamiento al concepto de creencias epistemológicas se ofrece a continuación:

Inguanzo (2010) las define como conjunto de ideas personales dinámicas y no verificables que pueden tener los profesores sobre la enseñanza, el aprendizaje y la naturaleza del conocimiento; estas ideas pueden regir su comportamiento, las decisiones que toman en el aula y la manera en que se relacionan con los alumnos. (p. 35).

Esta definición es relevante ya que propone no solamente las creencias epistemológicas del profesor sobre la naturaleza del conocimiento y el aprendizaje, sino que incluye aquellas que este posee sobre la enseñanza.

Aunque algunos autores las separan como creencias independientes, otros (Ching \& Swe, 2008) consideran que las creencias epistemológicas y las creencias de la enseñanza o pedagógicas son aquellas que poseen los individuos sobre la naturaleza del conocimiento y el aprendizaje y cómo estas categorías son cultivadas en clase. Se supone en esta concepción una relación muy estrecha entre ellas, por lo que, al hablar de las creencias epistemológicas de los profesores, se consideran las creencias sobre la enseñanza.

Las creencias de la enseñanza son aquellas consideraciones del profesor sobre lo que debe enseñar y cómo hacerlo donde se incluye la concepción de su rol de profesor, 
la metodología a utilizar y los recursos a emplear dentro de su clase (Azcarate \& Moreno, 2003). Además se debe tener en cuenta que el estilo de aprendizaje del profesor repercute en su forma de enseñar (Pintor \& Vizcarro, 2005).

Gonzalo Inguanzo propone evaluar las creencias de los profesores reconociendo la multidimensionalidad de las mismas, así como la asincronía que siguen en su desarrollo. Considera las dimensiones de Schommer (1994) y retoma otras propuestas, operacionalizando las creencias epistemológicas de los docentes en tres constructos fundamentales: creencias sobre la naturaleza del conocimiento, creencias sobre el aprendizaje y creencias sobre la enseñanza (Inguanzo, 2010).

Dentro de las creencias acerca del conocimiento incluye la utilidad y la naturaleza del conocimiento así como la fuente, la estructura, la estabilidad o certeza. Propone evaluar la dimensión "naturaleza del conocimiento" como abstracta y en oposición a esta el nivel concreto, que tiende a la ingenuidad.

Las dimensiones que aborda sobre el aprendizaje son: la habilidad para aprender, los estilos de procesamiento, la velocidad y la evaluación del aprendizaje. Los estilos de procesamiento son abordados en la literatura original como estrategias cognitivas para el procesamiento de la información, que se manifiestan como indicadores para evaluar el aprendizaje autorregulado de los alumnos (Castañeda \& Peñalosa, 2010). Se evalúan en dos polos, el convergente (nivel de poco desarrollo o de ingenuidad y el procesamiento divergente (nivel sofisticado).

La evaluación del aprendizaje como práctica de los profesores orientada por sus creencias ha sido una categoría poco reconocida o investigada en el marco de la epistemología personal, (Prieto, 2008). Inguanzo propone evaluar si el profesor cree que la evaluación debe ser por objetivos y normas claramente definidas o de forma más flexible adaptándola a los alumnos. También si esta evaluación debe considerar los patrones cuantificables o debe ser valorando un aprendizaje integral.

Un aspecto interesante en la propuesta de este autor para el estudio de las creencias lo constituye la inclusión de los aspectos didácticos de la enseñanza. Estos son: la planificación de la clase, las actividades en clase y la autopercepción de las habilidades de enseñanza. En cuanto a la planificación de la clase, la concibe como una tarea desarrollada por el profesor que incluye la definición de los objetivos de aprendizaje, la selección de contenidos, las decisiones metodológicas sobre las actividades y la evaluación.

En cuanto a las actividades desarrolladas en clases, la propuesta se orienta a evaluar la creencia en la docencia participativa, la creación de ambientes activos de aprendizaje que favorecen el pensamiento crítico de sus alumnos y no solo la elaboración de instrucciones, sino cómo estas facilitan el descubrimiento del conocimiento por los alumnos.

La autopercepción de las habilidades de enseñanza del profesor se evalúa a partir de las creencias de autoeficacia docente (Prieto, 2008) para mejorar sus estrategias de enseñanza. En el caso del profesor es mayor en los que se sienten mejor preparados para enfrentar su asignatura. Por tanto si el profesor cree que sus estrategias o 
habilidades para enseñar son insuficientes y su autoeficacia es menor, esto puede generar la necesidad de actualizarlas y mejorarlas.

A juicio de las autoras de este trabajo, la propuesta de Gonzalo Inguanzo en su concepción, integra aspectos esenciales descritos en este apartado y que han sido retomados en esta investigación, para desde la línea del estudio de las creencias en dominios académicos, contar con una herramienta que permita evaluar desde la perspectiva del profesor, posibles causas de las dificultades en el aprendizaje y la enseñanza de una materia en particular, en este caso la matemática.

\section{Creencias epistemológicas en el profesor de matemática.}

De todas las disciplinas estudiadas en el campo de la epistemología personal, la matemática parece haber tenido una mayor atención en el estudio de las creencias epistemológicas en disciplinas específicas (Hofer, 2006). Se han estudiado las creencias epistemológicas de los profesores de matemática (Gil \& Rico, 2003; Gómez \& Silas, 2012; Schommer-Aikins, 2008) ya que estas, sean generales o específicas, no sólo afectan el desempeño de los alumnos sino también el de los docentes.

En un estudio comparativo con estudiantes y profesores de matemática de secundaria básica se demostró que tanto las creencias epistemológicas de los estudiantes como de los profesores tendían a ser ingenuas, resultado contradictorio que ha provocado inquietudes de los investigadores ya que se esperaba creencias más desarrolladas en los docente (Gómez \& Silas, 2012).

En la investigación de Azcarate y Moreno (2003) sobre las concepciones y creencias de un grupo de 6 profesores universitarios de matemática sobre la enseñanza de ecuaciones estructurales, se reflejó que los profesores apuntaban a concebir al aprendiz como ejecutor mecánico, que aprende por imitación, considerándolo un receptor pasivo del conocimiento matemático. Apoyándose en estas creencias, los profesores justificaban todas las decisiones de planificación, elección de contenidos y tareas de aprendizaje.

El contexto cubano no ha estado exento de la creencia sobre la matemática como disciplina científica compleja, en la que los resultados docentes no siempre son los más satisfactorios, por lo que se ha planteado la necesidad de profundizar en variables e indicadores que al estudiarlos conduzcan a innovaciones que aseguren elevar los indicadores de calidad de este proceso educativo. Se reconoce el estudio de las creencias epistemológicas sobre la matemática, tanto en alumnos como en los profesores como una alternativa para ello.

Los antecedentes en el estudio de las creencias en nuestro país son escasos, solo cuentan las investigaciones de Aguilar (2012); Mendoza (2012); Morell (2011) y Pulido (2012), en ningún caso se han enfocado a las creencias de los profesores según el dominio académico, por lo que no se cuenta con herramientas de evaluación concebidas para tales fines. El presente trabajo es un intento de ofrecer a la comunidad 
científica, un instrumento que permita estudiar las creencias epistemológicas de los docentes de secundaria básica en un dominio académico específico, la matemática.

El Cuestionario de creencias docentes diseñado por Inguanzo (2010), permite evaluar las creencias de los profesores en sentido general, está elaborado desde la perspectiva del pensamiento del profesor; por lo que se toma como instrumento para realizar su adaptación en función de que permita evaluar las creencias epistemológicas sobre la matemática de los profesores de esta asignatura en la secundaria básica, siendo este nuestro objetivo fundamental. Se comprueban sus propiedades psicométricas, se interpretan los resultados y se ofrecen conclusiones orientadoras para la futura intervención educativa.

El procedimiento de adaptación aquí mostrado se inscribe entre los grandes retos actuales que tiene el estudio de las creencias, definiéndose como necesidad crear instrumentos que permitan una manera objetiva de su medición (Schommer-Aikins, Beuchat-Reichardt, \& Hernández-Pina, 2012).

\section{Método}

\section{Participantes}

Participaron 17 especialistas que ofrecieron sus juicios valorativos con vistas a la validez de contenido. De ellos, el 70,6 \% han tenido aproximaciones al constructo creencias epistemológicas y el 29.4 \% son especialistas en validación de instrumentos y del contexto de la enseñanza de la matemática.

Los participantes en el pilotaje fueron 30 profesores de matemática de Secundaria Básica pertenecientes a la provincia de Sancti Spíritus, en un total de 5 escuelas. El $66.7 \%$ pertenecen al sexo femenino y el $33.3 \%$ al masculino y una media de edad de 38.4 años $(\mathrm{DT}=14.0)$. En cuanto a la formación profesional, el $83.3 \%$ es Licenciado en Matemática, 8.3 \% Licenciado en Ciencias Exactas, 4.2 \% Licenciado en Física y otro $4.2 \%$ es Profesor General Integral.

La muestra de profesores para responder al cuestionario modificado y para el análisis de sus propiedades psicométricas fue de 171 docentes pertenecientes a los territorios de Camagüey, Ciego de Ávila, Cienfuegos y Villa Clara. El 52.2\% son del sexo femenino y el $42.8 \%$ del masculino. El rango de edad se encuentra entre 20 y 73 años $(\mathrm{M}=35.4$; DT= 11.1).

\section{Instrumento}

Cuestionario de creencias epistemológicas sobre la matemática para profesores de secundaria básica, versión definitiva después del procedimiento de adaptación del Cuestionario de creencias docentes diseñado por Inguanzo (2010). Contiene 50 ítems, que son contestados a partir de una escala de Likert de 5 opciones, donde 1 es Completamente de acuerdo y 5 Completamente en desacuerdo. Pretende medir las 
creencias sobre la matemática a través de 3 constructos fundamentales, los cuales se describen a continuación con sus dimensiones e indicadores.

a) Constructo: Creencias sobre el conocimiento.

Dimensiones: Estructura del conocimiento (simple y aislado; complejo e integrado); Estabilidad del conocimiento (absoluto, cierto y estático; tentativo, relativo y dinámico); Fuente del conocimiento (la autoridad; construcción individual; cuestionable o incuestionable); Utilidad del conocimiento (aplicabilidadtransferibilidad; no aplicabilidad-no transferibilidad); Naturaleza del conocimiento (concreta o abstracta; proviene de evidencias científicas o del sentido común).

b) Constructo: Creencias sobre el aprendizaje.

Dimensiones: Habilidad para aprender (esfuerzo y no necesita cualidades innatas; cualidades innatas); Velocidad con la que ocurre (rápida o es un proceso gradual, sistemático); Estilo de procesamiento (convergente o divergente); Evaluación del aprendizaje (por criterio o por normas; calificación como lo más importante o valoración integral).

c) Constructo: Creencias sobre la enseñanza.

Dimensiones: Planificación de la clase (participación conjunta o participación única del profesor; claridad de los objetivos como lo más importante o claridad en los objetivos como uno de los aspectos); Actividades en clase (trabajo individual o trabajo en equipo; ofrece valoraciones sistemáticas a los alumnos, o no retroalimenta; solo en función de las orientaciones- instrucciones del profesor o se promueve la construcción personal); Autopercepción de habilidades para enseñar (autoeficacia del profesor, necesidad de actualizarse o no necesita más actualización).

\section{Procedimientos}

Se realizaron todos los trámites legales que conlleva la adaptación de instrumentos de una cultura a otra (entre ellos, autorización de su autor principal). Se procedió a la consulta a los especialistas para determinar las modificaciones a realizar afín de que el cuestionario evaluara el constructo creencias epistemológicas sobre la matemática de profesores de esta asignatura en la secundaria básica. Obteniéndose la primera versión del instrumento. Posteriormente se realizó el estudio piloto, a partir del cual el instrumento sufre otras modificaciones. Finalmente se administra el instrumento revisado después del juicio de especialistas y el pilotaje a una muestra mayor de profesores para el análisis de sus propiedades psicométricas.

Se solicitó la disposición y consentimiento para participar en el estudio a los centros educativos seleccionados de la región central del país, especialmente a directivos, profesores y metodólogos de la asignatura de matemática, obteniéndose en todos los casos la aprobación. El cuestionario se aplicó de forma individual. Los escenarios de aplicación fueron las reuniones de preparación metodológica, los departamentos docentes y aulas donde se encontraban los profesores. 
Se realizó el análisis de fiabilidad del instrumento y de la estructura factorial mediante el método de componentes principales, a través del SPSS versión 20.0, procedimiento que ha sido aplicado por la mayoría de los investigadores en esta línea de estudio (Levin \& Wadmany, 2006; Shommer, 1994; Schraw et al., 2002;Walker, 2007; Tang, 2010).

\section{Resultados}

\section{Resultados del criterio de expertos}

Los especialistas consideraron que: de los 50 ítems, 44, (el 88 \%), medían el constructo, alcanzándose valores de 5 ("completamente"), de 4 ("en parte considerable") y de 3 ("en parte"). Solamente 4 ítems fueron considerados como que medían el constructo en una "mínima parte” y 2 ítems "en nada”.

Con los criterios de los especialistas se modificó el contenido de algunos indicadores e ítems. Dichas modificaciones pretendían ajustar el instrumento al dominio académico de la matemática.

\section{Modificaciones al cuestionario en la presentación de la matriz}

Con el procedimiento de adaptación desarrollado por las autoras del presente estudio, la matriz original sufre algunas modificaciones concernientes a la línea del estudio de las creencias en dominios específicos.

Así por ejemplo en la dimensión "estructura del conocimiento", en el instrumento original se reflejaban como indicadores Aislado-Integrado; siguiendo la propuesta de Shommer (1994), el análisis de las creencias sobre la estructura del conocimiento implica la evaluación en los términos: el conocimiento es Simple o Complejo, por lo que integramos estas dos propuestas. La dimensión queda definitivamente así: Constructo: Creencias sobre el conocimiento. Dimensión Estructura. Indicadores Aislado-Simple (ítems: 29- Los contenidos de la Matemática deben ser independientes uno de otros); Integrado-Complejo (ítems: 33.- Los contenidos de la Matemática son interdependientes; 20- Considero que el alumno aprende mejor si le pido integrar los contenidos).

En la dimensión "estabilidad del conocimiento", se agruparon de manera diferente los indicadores. En la versión original del cuestionario estaban organizados de la forma "cierto-tentativa” y "estático-dinámica”, se modifican quedando planteados "ciertoestático” y "tentativo-dinámico”.

Se modifican los indicadores de la dimensión "utilidad del conocimiento", que estaban presentados de la forma "no transferible-transferible" y "visión actual-visión futura". Se integran la aplicabilidad, transferibilidad y la visión futura del conocimiento en un solo indicador; y no aplicabilidad- no transferibilidad- visión actual en otro. 
En el constructo “creencias de la enseñanza”, se modificó la dimensión "planificación de la clase”, estaba presentada de la siguiente forma: “planeo-no planeo"; “claridad en los objetivos-sin claridad en los objetivos”. En los criterios de los especialistas este último indicador resultaba confuso por lo que se propone que se modifique de la siguiente forma "claridad en los objetivos como lo más importante de la planeación- claridad de los objetivos como uno de los aspectos de la planeación”.

Dentro del constructo “creencias del aprendizaje”, en la dimensión "habilidad para aprender" los indicadores estaban combinados "esfuerzo-sin esfuerzo"; habilidad innata-no innata”, quedando planteados “esfuerzo-aprendida y "sin esfuerzohabilidades innatas”, siguiendo el mismo principio que las dimensiones anteriores.

\section{Modificaciones a la escala}

En el instrumento propuesto por Inguanzo (2010) la escala tenía cuatro opciones. Los especialistas en la construcción de cuestionarios que participaron en el estudio, recomendaron que la escala tuviera un número impar de opciones, de modo que el punto medio fuera algo neutral y a ambos lados se tuviera desde el extremo positivo hasta el negativo. Se modificó dicha escala quedando de la siguiente forma: 1Completamente de acuerdo; 2-De acuerdo; 3-Neutral; 4-En desacuerdo y 5Completamente en desacuerdo. También se realizaron modificaciones a las instrucciones, las que fueron propuestas por los lingüistas.

\section{Principales modificaciones a los ítems}

Se agrega la palabra “Matemática” a los ítems del 1 al 4, del 6 al 10, el 12 y el 13, del 15 al 19, el 21, 22, 24, 28, 29, 31, del 33 al 44, el 46, 48 y 49. Esta propuesta de los especialistas permitió ajustar el instrumento a utilizar en función del dominio académico de la matemática. Se agregó “secundaria básica” a los ítems 23, 41 y 3 para ubicarlo en el contexto de desempeño de los profesores objeto de estudio.

\section{Modificaciones después del estudio piloto.}

Se realizaron cuando el 50 \% más 1 realizó la propuesta de cambio. Se agruparon en los siguientes criterios: términos de difícil comprensión (por ejemplo en el ítem 1); palabras con significados diferentes para el contexto mexicano (donde fue diseñado el instrumento) y el escenario cubano (por ejemplo el ítem 15, palabra "invariantes" por “inmodificable”); en el ítem 22 se sustituye "pericia” por "dominio”; "programas de estudio” por la frase “programa de la Matemática de la Secundaria Básica” (ítem 23); se propone cambiar "conferencia” por "la clase de nuevo contenido" (ítem 44) por ajustarse más al contexto cubano. Por solo citar algunos cambios.

Después de este procedimiento se obtuvo como resultado la tercera versión y final del instrumento. 


\section{Análisis de las propiedades psicométricas}

El coeficiente de consistencia interna o Alfa de Crombach alcanzó un valor de .75. Este coeficiente indica que la consistencia del instrumento tiende a ser aceptable a partir de los criterios encontrados en la literatura (Hernández, Fernández-Collado, \& Baptista, 2006).

El cálculo del KMO (Medida de adecuación muestral de Kaiser-Meyer-Olkin) marcó una puntuación de .83 por lo que se considera que la idea del análisis factorial es positiva y viable siendo mayor que .75 .

Dentro de la matriz, quedan como resultado 3 factores, que explican el $68 \%$ de la varianza. En la siguiente tabla se ilustran los ítems que cargan en cada uno de ellos con sus respectivas saturaciones.

\begin{tabular}{|c|c|c|c|}
\hline \multirow[b]{2}{*}{ Ítems } & \multicolumn{3}{|c|}{ Factores } \\
\hline & I & II & III \\
\hline $\begin{array}{l}\text { 1) Los estudiantes disfrutan más las clases de matemática cuando los } \\
\text { objetivos e indicadores de evaluación han sido claramente definidos }\end{array}$ & .71 & & \\
\hline $\begin{array}{l}\text { 3) El alumno de secundaria que es lento para aprender la matemática no } \\
\text { podrá cambiar su ritmo de aprendizaje. }\end{array}$ & .61 & & \\
\hline $\begin{array}{l}\text { 9) Los contenidos relacionados con la matemática son concretos y facilitan } \\
\text { entender problemáticas específicas. }\end{array}$ & .68 & & \\
\hline $\begin{array}{l}\text { 10) Considero que si el alumno no entiende algo en matemática es difícil } \\
\text { que lo aprenda aunque se esfuerce. }\end{array}$ & .78 & & \\
\hline $\begin{array}{l}\text { 12) Los conocimientos adquiridos en la matemática permiten explicar } \\
\text { muchos de los fenómenos que se presentan en la vida diaria. }\end{array}$ & .87 & & \\
\hline $\begin{array}{l}\text { 17) El buen profesor de matemática debe usar métodos de enseñanza que } \\
\text { maximizan la interacción profesor - alumno y alumno- alumno. }\end{array}$ & .73 & & \\
\hline 20) Considero que el alumno aprende mejor si le pido integrar los & .73 & & \\
\hline $\begin{array}{l}\text { 21) Los contenidos relacionados con la matemática son abstractos. } \\
\text { 21nitenins. }\end{array}$ & -.71 & & \\
\hline $\begin{array}{l}\text { 22) El buen profesor de matemática debe demostrar su dominio en los } \\
\text { contenidos disciplinares antes de pedirlo en sus alumnos. }\end{array}$ & .89 & & \\
\hline $\begin{array}{l}\text { 27) Una parte importante de la enseñanza de la matemática es saber } \\
\text { evaluar el aprendizaje de los alumnos. }\end{array}$ & .89 & & \\
\hline 33) Los contenidos de la matemática están interrelacionados. & .89 & & \\
\hline $\begin{array}{l}\text { 35) Lo importante de aprender los contenidos de la matemática es } \\
\text { conseguir buenas calificaciones. }\end{array}$ & .72 & & \\
\hline 37) Lo más importante de la planificación de la clase de matemática es que & & & \\
\hline $\begin{array}{l}\text { los objetivos de ésta reflejen las motivaciones de los involucrados en el } \\
\text { proceso. }\end{array}$ & .78 & & \\
\hline $\begin{array}{l}\text { 45) Considero que el alumno que ha tenido dificultades para aprender } \\
\text { siempre las tendrá. }\end{array}$ & .74 & & \\
\hline $\begin{array}{l}\text { 47) En un ambiente de aprendizaje integral las calificaciones son } \\
\text { inapropiadas. }\end{array}$ & -.71 & & \\
\hline $\begin{array}{l}\text { 16) La explicación teórica que sustenta los contenidos de la matemática es } \\
\text { cierta }\end{array}$ & & .55 & \\
\hline
\end{tabular}


34) Es recomendable que el profesor no ofrezca en las clases

retroalimentación a sus alumnos.

42) El buen profesor de matemática debe poner exámenes de manera sistemática.

25) A los estudiantes se les deberían dar oportunidades de participar activamente en la selección de los temas que se verán en la clase.

38) El buen profesor de matemática considera que el aprendizaje se logra

en condiciones donde él dice lo que hay que hacer.

Tabla 1. Factores principales generados del Análisis Factorial del Cuestionario de creencias epistemológicas sobre la matemática.

El factor 1 evalúa las creencias que tienen los profesores sobre el conocimiento, la enseñanza y el aprendizaje. En las dimensiones que pertenecen a los constructos creencias sobre el conocimiento y creencias sobre el aprendizaje se denota la asincronía en el desarrollo del sistema de creencias epistemológicas, se manifiestan ambos polos de desarrollo (ingenuidad-poco desarrollo y sofisticado), así por ejemplo las creencias sobre el conocimiento, en las que se expresan las dimensiones: Estructura, Utilidad, Naturaleza y Fuente, las tres primeras expresan este desarrollo irregular: los profesores creen en la complejidad, utilidad y nivel abstracto del conocimiento matemático pero a la misma vez lo conciben como fragmentado, que lo importante de adquirirlo se ciñe a la práctica escolarizada. La dimensión Fuente, clarifica una postura en el docente en la que él se concibe como autoridad, portador de los contenidos a aprender.

Las creencias sobre el aprendizaje manifiestan niveles de ingenuidad, al concebir que la habilidad para aprender requiere de un componente innato y que por mucho que los alumnos se esfuercen, aquel que ha tenido dificultades para aprender siempre las tendrá. No obstante, en este mismo factor se identificó que las creencias sobre la enseñanza, en los profesores del estudio presenta un nivel sofisticado, al concebir en la planificación de la clase la definición de los objetivos como uno de los aspectos del proceso, lo que nos hace suponer que reconocen otros que también determinan la calidad de esta práctica, igual comportamiento se encontró en la dimensión actividades en clase, al concebirla desde la organización del trabajo en equipo. En la Tabla 2 se muestran los análisis descriptivos de las dimensiones pertenecientes a este factor, el valor de la media más bajo fue de 1.92 y el más alto 3.37. La asimetría de la distribución de puntuaciones es mayor que 1 en las dimensiones: Fuente, Utilidad y Actividades en clases, es negativa en Naturaleza, Habilidad y Velocidad tendiendo a los valores más altos de la escala. Las correlaciones entre las dimensiones y el factor son altamente significativas. 


\begin{tabular}{lccccc}
\hline $\begin{array}{l}\text { Dimensiones/ N= } \\
\mathbf{1 7 1}\end{array}$ & $\mathbf{M}$ & $\mathbf{D T}$ & As & $\mathbf{K}$ & $\mathbf{r}$ \\
\hline Estructura & 2.68 & 0.70 & 0.02 & 0.36 & $.472^{* *}$ \\
Fuente & 1.92 & 1.40 & 1.22 & 0.00 & $.624^{* *}$ \\
Utilidad & 2.67 & 0.77 & 1.51 & 12.6 & $.433^{* *}$ \\
Naturaleza & 2.79 & 0.77 & -.156 & 0.55 & $.344^{* *}$ \\
Planificación & 2.14 & 1.29 & .890 & -0.50 & $.591^{* *}$ \\
Actividades Clase & 2.08 & 1.59 & 1.05 & -0.54 & $.697^{* *}$ \\
Habilidad aprender & 3.37 & 1.34 & -.569 & -1.08 & $-.208^{* *}$ \\
Velocidad & 3.10 & 1.16 & -.342 & -1.08 & -.071 \\
Evaluación & 2.65 & 0.80 & .312 & 0.83 & $.482^{* *}$ \\
\hline
\end{tabular}

Tabla 2. Estadísticos descriptivos Factor I

El factor 2 evalúa la estabilidad del conocimiento; las creencias sobre las actividades en clase y la evaluación del aprendizaje. Informa sobre la creencia en los profesores en la certeza del conocimiento así como de una concepción de la evaluación según normas y criterios definidos con anterioridad, además de no retroalimentar al alumno durante las actividades escolares. La Tabla 3 muestra que el valor más bajo de la media se encuentra en la Estabilidad y el más alto en Actividades en clase, las correlaciones también son altamente significativas.

\begin{tabular}{lccccc}
\hline Dimensiones/ N=171 & M & DT & As & K & r \\
\hline Estabilidad & 1.52 & 0.81 & 2.06 & 5.24 & $.504^{* *}$ \\
Actividades clase & 3.95 & 1.17 & -1.13 & 0.45 & $.361^{* *}$ \\
Evaluación & 2.05 & 1.87 & 7.38 & 76.1 & $.785^{* *}$ \\
\hline
\end{tabular}

Tabla 3. Estadísticos descriptivos Factor II

El factor 3 se considera como planificación compartida pero con autoridad docente. Está compuesto por ítems que señalan (“A los estudiantes se les deberían dar oportunidades de participar activamente en la selección de los temas que se verán en la clase"), por otro lado "El buen profesor de matemática considera que el aprendizaje se logra en condiciones donde él dice lo que hay que hacer". Nótese que en ambas dimensiones (ver Tabla 4) el valor de la asimetría es negativa y las correlaciones son igualmente significativas.

\begin{tabular}{lccccc}
\hline Dimensiones/ N= 171 & M & DT & As & K & r \\
\hline Planificación Clase & 3.11 & 1.37 & -.175 & -1.29 & $.814^{* *}$ \\
Fuente & 3.66 & 1.18 & -.860 & -.187 & $.736^{* *}$ \\
\hline
\end{tabular}

Tabla 4. Estadísticos descriptivos Factor III 
El análisis de las dimensiones de concepciones epistemológicas del Cuestionario de Creencias Epistemológicas sobre la matemática, atendiendo sólo a la estructura factorial identificada indica lo siguiente. En cuanto a la homogeneidad y heterogeneidad de cada grupo o factor, se observa que las dimensiones estructura, utilidad, naturaleza y evaluación del factor I, presentan una mayor homogeneidad entre sí comparada con las dimensiones Fuente; Planificación; Actividades en Clase; Habilidad para aprender y Velocidad de ese mismo factor. La dificultad en tener un grupo más homogéneo con un pensamiento sofisticado en la estructura del conocimiento, del aprendizaje y la enseñanza de la matemática es más difícil, porque implica una reflexión profunda y un quehacer sustentado en una posición sofisticada con respecto a la esencia de este conocimiento.

La presencia simultánea de creencias, en este caso ingenua y sofisticada frente al conocimiento, el aprendizaje y la enseñanza se observó en el análisis realizado con las dimensiones del Cuestionario. Se demuestra, tal como lo plantea Schommer-Aikins (2008), que las creencias no se desarrollan de la misma manera. El punto más importante es que uno simplemente no puede asumir que las creencias epistemológicas estén sincronizadas (Duell \& Schommer-Aikins, 2001). Este supuesto ha sido corroborado en este estudio, las creencias sobre la matemática de los profesores participantes no presentan un único nivel de desarrollo, las propiedades psicométricas del instrumento adaptado mostraron índices de fiabilidad y validez que tienden a ser aceptables.

\section{Conclusiones}

En este trabajo se concretó la adaptación y análisis de las propiedades psicométricas de un cuestionario con el cual medir las creencias epistemológicas sobre la matemática de los profesores que imparten esta asignatura en la enseñanza secundaria. Ante los resultados obtenidos se asume, siguiendo la línea de Muis (2004); Op't Eynde, de Corte y Verschaffel (2006); Steiner (2007); Tang (2010) y Walker (2007) que el sistema de creencias sobre la matemática, de los profesores que imparten esta asignatura en la secundaria es multifactorial.

Desde el análisis factorial exploratorio los tres factores cumplen los requisitos psicométricos necesarios, por lo que se puede concluir que el Cuestionario de creencias epistemológicas sobre la matemática de profesores de esta asignatura en la secundaria básica mide de forma válida y fiable tres factores del sistema de creencias, el cual muestra niveles asincrónicos en su desarrollo.

En estudios donde se ha analizado la creencia sobre la fuente del conocimiento en profesores de Matemática de secundaria básica (Gómez \& Silas, 2012), estos han manifestado creencias fuertemente arraigadas acerca de sí mismos como esa fuente de donde procede el conocimiento, preponderando este nivel de desarrollo ingenuo sobre la consideración del conocimiento construido por parte del alumno, lo cual al no ser estimulado influye en que los estudiantes simplifiquen más sus respuestas en determinados exámenes, ya que solo responderán de la manera enseñada por el 
profesor y limitará su creatividad. Este resultado se corresponde con los encontrados en el presente trabajo, si bien esto demuestra la réplica del estudio pese a las diferencias culturales, estas consideraciones de los docentes que apuntan a una enseñanza centrada en el rol docente podrían ir en detrimento del necesario control que deben ejercer los alumnos sobre la construcción del conocimiento.

En cuanto a las creencias sobre la enseñanza los resultados apuntan a que los docentes creen que en las actividades en clase deben hacer uso de métodos como el trabajo en equipo, ya que contribuye mejor al aprendizaje de los alumnos, lo cual es un aspecto positivo y que tiende al pensamiento sofisticado. Este tipo de método de enseñanza responde a una perspectiva constructivista (Aguilar, 2012; Chan, 2005; Hofer, 2001).

Estos resultados se pueden tomar como referentes para el trabajo metodológico, en el que se puede abordar las creencias sobre las actividades en clase, pues aunque reconocen la importancia del trabajo en equipo, este no se utiliza frecuentemente en las clases, pues muchas veces el profesor aunque actúa según sus concepciones no llega a poner en práctica todo su sistema de creencias. En cuanto a los objetivos se debe insistir en que el profesor no solo se oriente a los mismos sino que también preste atención a otros aspectos de la clase que también son importantes como los propios métodos, la evaluación, la comunicación educativa, los contenidos, la participación de los alumnos entre otros.

Aunque los profesores creen que el aprendizaje debe evaluarse a través de normas establecidas, reconocen la importancia de la evaluación integral, lo que coincide con las ideas de Vila y Callejo (2004) cuando fundamentan que la evaluación en el aprendizaje de la Matemática, ya sea explícita o implícitamente, va modelando creencias en los alumnos acerca de lo que es más o menos importante para su aprendizaje.

Los resultados encontrados apuntan a la creencia en el profesor de que el estilo de procesamiento que garantiza el aprendizaje es el divergente. Este estilo contribuye al desarrollo del pensamiento creativo, lo que beneficia tareas como la resolución de problemas.

De manera general, los factores encontrados en el presente estudio, mostraron evidencias de diferentes niveles de desarrollo del sistema de creencias de los profesores participantes, acerca de la naturaleza del conocimiento, del aprendizaje y la enseñanza de la Matemática. Algunas pueden contribuir a desarrollar una práctica educativa de mejor calidad como son las creencias sobre la importancia del método del trabajo en equipo; la concepción del conocimiento matemático como abstracto, estructurado, complejo, aplicable y transferible; las creencias sobre el aprendizaje gradual, sistemático y la concepción de una evaluación integral del mismo, vistas en el primer factor, así como la creencia en el estilo de procesamiento divergente, la docencia participativa, que se encuentran en los restantes factores. Sin embargo se han identificado juntamente con ellas otro set de creencias pertenecientes a la epistemología personal de los profesores que evidencian un pensamiento 
epistemológico ingenuo en relación al conocimiento, enseñanza y aprendizaje de la matemática.

Se necesita ir consolidando una línea de investigación en las instituciones responsables de la superación de los docentes, que contribuya a la evaluación y desarrollo de un pensamiento sofisticado relacionado con los procesos de enseñanzaaprendizaje de la matemática. Del análisis de las propiedades psicométricas del instrumento utilizado en este estudio surgen otras interrogantes ¿en qué medida la experiencia como docentes de matemática en el nivel de secundaria beneficia la construcción de un pensamiento sofisticado? o ¿Qué factores inciden en un cambio de pensamiento respecto al conocimiento, el aprendizaje y la enseñanza de esta materia en los docentes encuestados? La preocupación por el perfeccionamiento docente es algo vigente y las creencias son a nuestro juicio un terreno sobre el cual incidir.

\section{Referencias bibliográficas}

AGUILAR, T. (2012). Creencias epistemológicas del conocimiento en profesores de preuniversitario. Tesis de grado, Universidad de La Habana, Ciudad Habana.

AZCARATE, C., \& MORENO, M. (2003). Concepciones y creencias de los profesores universitarios de matemáticas acerca de la enseñanza de las ecuaciones diferenciales. Enseñanza de la ciencia, 2, 265-280.

BRATEN, I., \& STROMSO, H. I. (2005). The relationship between epistemological beliefs implicit theories of intelligence and self-regulated learning among Norwegian postsecondary students. British Journal of Educational Psychology, 75, 57-62.

CANO, F. (2005). Epistemological beliefs and approaches to learning: their change through secondary school and their influence on academic performance. British Journal of Educational Psychology, 75, 203-221.

CASTAÑEDA, S., \& PEÑALOSA, E. (2010). Validando constructos en epistemología personal. Revista Mexicana de Psicología, 27(1), 65-75.

CHAN, W. (2005). Impact of Constructivist Teaching on Students'Beliefs about Teaching and Learning in Introductory Physics. Canadian Journal of Science, Mathematics and Technology Education, 95-109.

CHING, C., \& SWE, M. (2008). Assessing the Epistemological and Pedagogical Beliefs Among Pre-service Teachers in Singapore. In M. S. Khine (Ed.), Knowing, Knowledge and Beliefs Epistemological Studies across Diverse Cultures (pp. 287299). Australia: Springer.

CONLEY, A., \& HARRISON, D. (2004). Changes in epistemological beliefs in elementary science studentes. Contemporary Educational Psychology, 186-204.

DE CORTE, E., OP’T EYNDE, P., \& VERSCHAFFEL, L. (2002). Knowing what to believe: The relevance of students' mathematical beliefs for mathematics education. 
In B. K. Hofer, Personal epistemology. The psychology of beliefs about knowledge and knowing (pp. 297-320). Mahwah NJ: Lawrence Erlbaum.

DUELL, O., \& SCHOMMER-AIKINS, M. (2001). Measures of People's Beliefs About Knowledge and Learning. Educational Psychology Review, 13(4), 419-449.

GARCÍA, M., \& C., S. (2011). Creencias Epistemológicas de Estudiantes de Pedagogía en Educación Parvularia, Básica y Media: ¿Diferencias en la Formación Inicial Docente? PSYKHE, 20, 29-43.

GIL, F., \& RICO, L. (2003). Concepciones y creencias del profesorado de secundaria sobre enseñanza y aprendizaje de las matemáticas. Enseñanza de las ciencias, 21(1), 27-47.

GÓMEZ, L., \& SILAS, C. (2012). Las creencias epistemológicas de alumnos y profesores de $1^{\circ}$ de secundaria. Diálogos sobre Educación, 3(5), 1-14.

HAMMER, D., \& ELBY, A. (2002). On the form of a personal epistemology. En B. K. Hofer, \& P. Pintrich (Edits.), Personal epistemology: The psychology of beliefs about knowledge and knowing (págs. 169-190). Mahwah, NJ: Erlbaum.

HERNÁNDEZ, R., FERNÁNDEZ-COLLADO, C., \& BAPTISTA, P. (2006). Metodología de la Investigación. México: Mc Graw Hill.

HOFER, B. (2001). Personal Epistemology Research: Implications for Learning and Teaching. Journal of Educational Psychology Review, 13, 353-383.

HOFER, B. (2004). Exploring the dimensions of personal epistemology diferining classroom contexts: Student interprtetions during the first year of college. Contemporary Educational Psychology, 24, 129-163.

HOFER, B. (2006). Domain specificity of personal epistemology: Resolved questions, persistent issues, new models. International Journal of Educational Research, 45, 85-95.

HOFER, B. (2008). Personal Epistemology and Culture. Knowing, Knowledge and Beliefs. Epistemological Studies across Diverse Cultures. Australia: Springer.

HOFER, B., \& PINTRICH, P. (1997). The development of epistemological theories: Beliefs about knowledge and knowing and their relation to learning. Review of Educational Research, 67(1), 88-140.

INGUANZO, G. (2010). Creencias de los profesores de nivel de licenciatura sobre la naturaleza del conocimiento y los procesos de enseñanza y aprendizaje. Tesis Doctoral, Universidad Iberoamericana de Puebla, Puebla.

LEVIN, T., \& WADMANY, R. (2006). Teachers' Beliefs and Practices in Technology in Education. Journal of Research on Technology in Education, 39, 157-181.

MANSON, L. (2004). Paul R. Pintrich y la investigación sobre creencias epistemológicas. Revista Electrónica de Investigación Psicoeducativa, 2, 163-170. 
MENDOZA, Z. (2012). Caracterización de las creencias epistemológicas sobre la matemática y el rendimiento académico de estudiantes de 8vo grado del municipio de Santa Clara. Universidad Central de Las Villas. Santa Clara: Feijoó.

MORELL, M. (2011). Creencias Epistemológicas, Estrategias Metacognitivas y Rendimiento Académico en estudiantes de primer año de Ingeniería. Tesis de Maestría, Facultad de Psicología Universidad de La Habana, La Habana.

MUIS, K. (2004). Personal epistemology and mathematics: A criticalreview and synthesis of research. Review of Educational Research, 74, 317-377.

OP'T EYNDE, P., DE CORTE, E., \& VERSCHAFFEL, L. (2006). Epistemic dimensions of students' mathematics-related belief systems. International Journal of Educational Research, 45, 57-70.

PERRY, W. (1970). Forms of intellectual and ethical development in the college years: A scheme. New York: Holt.

PINTOR, M., \& VIZCARRO, C. (2005). Cómo aprenden los profesores. un estudio empírico basado en entrevistas. Revista Complutense de Educación, 16, 623-644.

PINTRICH, P. R. (2002). Future challenges and directions for theory and research on personal epistemology. En P. Pintrich (Ed.), Personal epistemology: The psychology of beliefs about knowledge and knowing (págs. 389-414). Mahwah, NJ, USA: Lawrence Erlbaum Associates.

PRIETO, M. (2008). Creencias de los profesores sobre evaluación y efectos incidentales. Revista de Pedagogía, 29.

PULIDO, M. (2012). Validación de constructo de la encuesta de Creencias Epistemológicas sobre la Matemática para alaumnos de la enseñanza media. Tesis de grado, Universidad Central de Las Villas, Santa Clara.

SCHOMMER, M. (1990). Effects of Beliefs about the Nature of Knowledge on Comprehension. Journal of Educational Psychology, 82, 498-504.

SHOMMER, M. (1994). Synthesizing Epistemological Belief Research: Tentative Understandings and Provocative Confusions. Educational Psychology Review, 6, 293-316.

SCHOMMER-AIKINS, M. (2008). Applying the Theory of an Epistemological Belief System to the Investigation of Students' and Professors'Mathematical Beliefs. In M. S. Khine (Ed.), Knowing, Knowledge and Beliefs Epistemological Studies across Diverse Cultures (pp. 303-323). Australia, Springer.

SCHOMMER-AIKINS, M., Beuchat-Reichardt, M., \& Hernández-Pina, F. (2012). Creencias epistemológicas y de aprendizaje en la formación inicial de profesores. Anales de Psicología, 28(2), 465-474.

SCHRAW, G., BENDIXEN, L., \& DUNKLE, M. (2002). Development and validation of the Epistemic Belief Inventory (EBI). In P. Pintrich (Ed.), Personal 
epistemology: The psychology of beliefs about knowledge and knowing (pp. 261276). Mahwah, NJ: Lawrence Erlbaum Associates.

SERRANO, R. (2010). Pensamientos del profesor: un acercamiento a las creencias y concepciones sobre el proceso de enseñanza-aprendizaje en la Educación Superior. Revista de Educación, 352, 267-287.

STEINER, L. (2007). The effect of personal and epistemological beliefs performance in a collage development al mathematics class. An abstract of a dissertation, Kansas State University, Manhattan.

TANG, J. (2010). Exploratory and confirmatory factor analysis of epistemological beliefs Questionary about mathematics for chinese junior middle school students. Journal of Mathematics Education, 3, 89-105.

VILA, A., \& CALLEJO, M. (2004). Matemáticas para aprender a apensar. El papel de las creencias en la resolución de problemas (Narcea ed.). Madrid.

WALKER, D. (2007). The development and constrct validation of the epistemological beliefs survey for Mathematics. Unpublished partial fulfillment of the requirements for the Degree for Doctor of philosophy, University of Oklahoma, Oklahoma.

\section{Correspondencia con los autores}

Annia Esther VIZCAINO ESCOBAR

Facultad de Psicología. Universidad Central "Marta Abreu” de Las Villas.

Carretera a Camajuaní \# 258 / Tomás Ruíz y Ana Pegudo. Santa Clara cp.

50100. Villa Clara. Cuba.

e-mail: annia@uclv.edu.cu 